PROCEEDINGS OF THE AMERICAN MATHEMATICAL SOCIETY

Volume 124, Number 12, December 1996, Pages 3703-3710

S 0002-9939(96)03467-3

\title{
THEOREM OF KURATOWSKI-SUSLIN FOR MEASURABLE MAPPINGS. II
}

\author{
ANDRZEJ WIŚNIEWSKI
}

(Communicated by Palle E. T. Jorgensen)

\begin{abstract}
The purpose of this paper is to describe these $\mu$-measurable mappings on a separable complete metric space with the Borel measure $\mu$, which transform every $\mu$-measurable set onto a $\mu$-measurable one. The obtained results are a generalization of the classical outcomes of Suslin and Kuratowski and the results from our previous paper.
\end{abstract}

\section{INTRODUCTION}

Let $X$ be a separable complete metric space and let $f$ be a one-to-one mapping on $X$. In his paper [4] Suslin proved that if $f$ is a continuous function, then for every Borel subset $B$ of $X$ the image $f(B)$ is also a Borel set. Kuratowski in [1] extended this theorem to the case of Borel mappings. Namely, he proved the following theorem.

Theorem of Kuratowski. Let $X$ be a separable complete metric space and $X_{1}$ a Borel subset of $X$. If $f$ is a one-to-one Borel measurable mapping from $X_{1}$ into $X$, then $f(B)$ is a Borel set for every Borel subset $B$ of $X_{1}$.

For details concerning the above theorem see also [2] (Chapter I.4).

In our previous paper [5] we investigated the possibility of the generalization of the above theorem of Suslin-Kuratowski to the case of measurability (of sets $B$ and $f(B)$ ) with respect to some measure on $X$ instead of their Borel measurability. It appears that such a generalization need not always be true, even in the case of the measurability with respect to the Lebesgue measure on a real line (see [5], Example 1) or in the case of translations in a linear space (see [5], Example 2). In [5] we have given the conditions under which the above mentioned generalization of the theorem of Suslin-Kuratowski is possible. Namely, it was shown that a one-toone Borel mapping $f$ on $X$ transforms every measurable set (with respect to some measure $\mu$ on $X$ ) onto a measurable one if and only if the measure $\mu$ is absolutely continuous with respect to the measure $\mu_{f}$ (an image of $\mu$ under the mapping $f$ ) ([5], Theorem 1).

Received by the editors November 28, 1994 and, in revised form, April 14, 1995.

1991 Mathematics Subject Classification. Primary 28A05, 28A20; Secondary 28C20, 60B05, 60B11.

Key words and phrases. Borel sets, measurable and non-measurable sets, Borel mappings, measurable mappings, absolute continuity of measures.

(C)1996 American Mathematical Society 
In the present paper we shall continue this investigation. Our purpose will be the further extension of the theorem of Suslin-Kuratowski. We generalize this theorem to the case of $\mu$-measurable mappings. Similarly as in the case of Borel mappings we give also the description of these $\mu$-measurable mappings on $X$ for which such a generalization is possible. Moreover, we prove some similar properties of $\mu$ measurable mappings, which may be treated as a specific form of the theorem of Suslin-Kuratowski, however concerning not images but inverse images of measurable sets.

Let $(X, \mathcal{B}, \mu)$ be a measure space and $(Y, \mathcal{F})$ a measurable space. Let $f$ be a measurable mapping from $X$ into $Y$. By $\mu_{f}$ we shall denote the image of $\mu$ under the mapping $f$, i.e. a measure on $\mathcal{F}$ defined by the formula $\mu_{f}(A)=\mu\left(f^{-1}(A)\right)$ for $A \in \mathcal{F}$.

A measure $\mu$ is said to be a non-atomic measure if $\mu(\{x\})=0$ for any $x \in X$ (provided that $\{x\} \in \mathcal{B}$ ).

Let $(X, \mathcal{B})$ be a measurable space and let $\mu$ and $\nu$ be two measures on $\mathcal{B}$. We say that the measure $\mu$ is absolutely continuous with respect to the measure $\nu$, and we write $\mu \ll \nu$ iff from $B \in \mathcal{B}$ and $\nu(B)=0$ it follows that $\mu(B)=0$. If at the same time $\mu \ll \nu$ and $\nu \ll \mu$, then we say that the measures $\mu$ and $\nu$ are equivalent, and we write $\mu \sim \nu$.

Let $(X, \mathcal{B}, \mu)$ be an arbitrary measure space. Denote by $\mathcal{B}_{\mu}$ the completion in measure $\mu$ of the $\sigma$-algebra $\mathcal{B}$, that is a $\sigma$-algebra of all subsets in $X$ of the form $B \cup N$, where $B \in \mathcal{B}$ and $N \subset A$ for some $A \in \mathcal{B}$ such that $\mu(A)=0$. The $\sigma$-algebra $\mathcal{B}_{\mu}$ is also called a $\sigma$-algebra of sets measurable with respect to the measure $\mu$, or a $\sigma$-algebra of $\mu$-measurable sets.

The measure $\mu$, which is defined on $\mathcal{B}$, we may in a natural way extend to a measure on $\mathcal{B}_{\mu}$ putting $\mu(B \cup N)=\mu(B)$.

For example, if $X=R$ is a real line, $\mathcal{B}=\mathcal{B}(X)$ is the Borel $\sigma$-algebra on $R$ and $\mu=m$ is the Lebesgue measure defined on $\mathcal{B}$, then $\mathcal{B}_{m}$ is a $\sigma$-algebra of sets measurable with respect to the Lebesgue measure (measurable in the sense of Lebesgue), and a measure $m$ extended to $\mathcal{B}_{m}$ is the classical Lebesgue measure on $R$.

It is well known that if $A$ is a Borel subset of $R$ with positive Lebesgue measure, then there exists a subset $B$ of $A$ which is non-measurable with respect to $m$. In this paper we will need some extension of this fact.

Lemma 1 ([5]). Let $X$ be a separable complete metric space and $\mathcal{B}=\mathcal{B}(X)$ the Borel $\sigma$-algebra on $X$. Suppose that $\mu$ is a $\sigma$-finite and non-atomic measure on $\mathcal{B}$. If $A \in \mathcal{B}$ and $\mu(A)>0$, then there exists a set $B \subset A$ such that $B \notin \mathcal{B}_{\mu}$ (i.e. $B$ is non-measurable with respect to $\mu$ ).

Let $(X, \mathcal{B}, \mu)$ be an arbitrary measure space, and $(Y, \mathcal{F})$ a measurable space. A mapping $f: X \rightarrow Y$ is called a $\mu$-measurable mapping if it is measurable with respect to $\left(\mathcal{B}_{\mu}, \mathcal{F}\right)$, i.e. if $f^{-1}(A) \in \mathcal{B}_{\mu}$ for each $A \in \mathcal{F}$.

Of course, every measurable mapping $f: X \rightarrow Y$ is $\mu$-measurable for any measure $\mu$ on $\mathcal{B}$.

\section{MAin Results}

In this section we shall deal with the generalization of the theorem of SuslinKuratowski to the case of $\mu$-measurable mappings. We give conditions under which such a generalization is possible. 
Throughout this section $X$ will always denote a separable complete metric space, $\mathcal{B}$ the Borel $\sigma$-algebra on $X$ and $\mu$ a Borel measure on $X$. As usual by $\mathcal{B}_{\mu}$ we shall denote the $\sigma$-algebra of $\mu$-measurable sets.

Now let $f$ be a $\mu$-measurable mapping from $X$ into $X$, i.e. for any $B \in$ $\mathcal{B}, f^{-1}(B) \in \mathcal{B}_{\mu}$.

In order to prove the main results of this paper we will need the following statement.

Lemma 2. For every $\mu$-measurable mapping $f: X \rightarrow X$ there exists a Borel subset $X_{0}$ of $X$ such that $\mu\left(X-X_{0}\right)=0$ and the restriction $\left.f\right|_{X_{0}}$ of the mapping $f$ to the set $X_{0}$ is Borel measurable.

Proof. It is clear that since $X$ is a separable metric space, then there exists a countable class $\Gamma=\left\{K_{n}, n=1,2, \ldots\right\}$ of Borel subsets of $X$ such that the Borel $\sigma$ algebra $\mathcal{B}$ is generated by this class, i.e. $\mathcal{B}=\sigma(\Gamma)(\sigma(\Gamma)$ denotes the least $\sigma$-algebra which contains $\Gamma$ ). Indeed, if $E$ is a countable dense set in $X$, then as a class $\Gamma$ we may take a set of all balls of radius $r$ with centre at the point $x$, where $r$ is an arbitrary rational number and $x$ is an arbitrary element of $E$.

Let $B_{n}=f^{-1}\left(K_{n}\right)$, for $n=1,2, \ldots$ Obviously $B_{n} \in \mathcal{B}_{\mu}$. Therefore $B_{n}=$ $A_{n} \cup C_{n}$, where $A_{n} \in \mathcal{B}, C_{n} \subset D_{n}, D_{n} \in \mathcal{B}$ and $\mu\left(D_{n}\right)=0$.

Put $D=\bigcup_{n=1}^{\infty} D_{n}$. Then $D \in \mathcal{B}$ and $\mu(D)=0$. Now let $X_{0}=X-D$. Then $X_{0}$ is a Borel subset of $X$ and $\mu\left(X-X_{0}\right)=0$.

Denote by $g$ the restriction of the mapping $f$ to the set $X_{0}$, i.e. $g=\left.f\right|_{X_{0}}$. We must show that $g$ is a Borel mapping. To prove this fact it is enough to show that $g^{-1}(B) \in \mathcal{B}$ for every set $B \in \Gamma$ (since $\sigma(\Gamma)=\mathcal{B}$ ), i.e. more precisely that for any $n=1,2, \ldots, g^{-1}\left(K_{n}\right) \in \mathcal{B}$.

Indeed, we have that $g^{-1}\left(K_{n}\right)=\left\{x \in X_{0}: g(x) \in K_{n}\right\}=\left\{x \in X_{0}: f(x) \in\right.$ $\left.K_{n}\right\}=f^{-1}\left(K_{n}\right) \cap X_{0}=B_{n} \cap X_{0}=\left(A_{n} \cup C_{n}\right) \cap X_{0}=\left(A_{n} \cap X_{0}\right) \cup\left(C_{n} \cap X_{0}\right)=$ $A_{n} \cap X_{0}$, since for any $n=1,2, \ldots, C_{n} \cap X_{0}=\varnothing$ (which follows from the fact that $\left.C_{n} \subset D_{n} \subset D=X-X_{0}\right)$. Therefore for any $n=1,2, \ldots, g^{-1}\left(K_{n}\right)=A_{n} \cap X_{0}$, and since $A_{n} \in \mathcal{B}$ and $X_{0} \in \mathcal{B}$, then $A_{n} \cap X_{0} \in \mathcal{B}$, i.e. $g^{-1}\left(K_{n}\right) \in \mathcal{B}$. This completes the proof.

Now we are ready to extend the results from [5], where the generalization of the theorem of Suslin-Kuratowski for Borel mappings was considered, to the case of $\mu$-measurable mappings.

Proposition 1. Let $f$ be a $\mu$-measurable and one-to-one mapping from $X$ into $X$. If $\mu \ll \mu_{f}$, then $f(B) \in \mathcal{B}_{\mu}$ for every set $B \in \mathcal{B}_{\mu}$.

Proof. In view of Lemma 2 there exists a Borel subset $X_{0}$ of $X$ such that $\mu\left(X-X_{0}\right)=0$ and the restriction of $f$ to $X_{0}$ is Borel measurable. Suppose that $\mu \ll \mu_{f}$ and let $B$ be an arbitrary $\mu$-measurable set, i.e. $B \in \mathcal{B}_{\mu}$.

Put $B_{1}=B \cap X_{0}$ and $B_{2}=B \cap\left(X-X_{0}\right)$. Then $B=B_{1} \cup B_{2}$, whence

$$
f(B)=f\left(B_{1}\right) \cup f\left(B_{2}\right) .
$$

First of all we show that $f\left(B_{1}\right) \in \mathcal{B}_{\mu}$. Since $B_{1} \in \mathcal{B}_{\mu}$ and $B_{1} \subset X_{0}$, then $B_{1}=A \cup N$, where $A \in \mathcal{B}$ and $A \subset X_{0}, N \subset A_{1}, A_{1} \in \mathcal{B}$ and $\mu\left(A_{1}\right)=0$. We have therefore that $f\left(B_{1}\right)=f(A) \cup f(N)$. Since $A$ is a Borel subset of $X_{0}$ and $\left.f\right|_{X_{0}}$ is Borel measurable, then by virtue of the Theorem of Kuratowski we infer that $f(A) \in \mathcal{B}$ and consequently $f(A) \in \mathcal{B}_{\mu}$. Note also that $f(N) \in \mathcal{B}_{\mu}$. Indeed, let $A_{0}=A_{1} \cap X_{0}$. Then $A_{0} \in \mathcal{B}$ and $A_{0} \subset X_{0}$, and applying again the Theorem 
of Kuratowski we get that $f\left(A_{0}\right) \in \mathcal{B}$. Moreover, $N \subset A_{1}$ and $N \subset B \subset X_{0}$, whence $N \subset A_{0}$. Since $f$ is an injection, we have that $f^{-1}\left(f\left(A_{0}\right)\right)=A_{0}$. Hence $\mu_{f}\left(f\left(A_{0}\right)\right)=\mu\left(f^{-1}\left(f\left(A_{0}\right)\right)\right)=\mu\left(A_{0}\right)$. But $\mu\left(A_{0}\right)=0$, since $A_{0} \subset A_{1}$ and $\mu\left(A_{1}\right)=$ 0 . Therefore $\mu_{f}\left(f\left(A_{0}\right)\right)=0$, whence by virtue of the assumption (i.e. $\mu \ll \mu_{f}$ ) we obtain that $\mu\left(f\left(A_{0}\right)\right)=0$. From $N \subset A_{0}$ it follows that $f(N) \subset f\left(A_{0}\right)$, which implies that $f(N) \in \mathcal{B}_{n}$. Hence, since also $f(A) \in \mathcal{B}_{\mu}$ and $f\left(B_{1}\right)=f(A) \cup f(N)$, we get that $f\left(B_{1}\right) \in \mathcal{B}_{\mu}$.

Now we show that $f\left(B_{2}\right) \in \mathcal{B}_{\mu}$. Note in the first place that since $X_{0} \in \mathcal{B}$ and $\left.f\right|_{X_{0}}$ is Borel measurable, then in view of the Theorem of Kuratowski $f\left(X_{0}\right) \in \mathcal{B}$. From $X=X_{0} \cup\left(X-X_{0}\right)$ it follows that $f(X)=f\left(X_{0}\right) \cup f\left(X-X_{0}\right)$, and since $f$ is a one-to-one mapping and $X_{0} \cap\left(X-X_{0}\right)=\varnothing$, then $f\left(X_{0}\right) \cap f\left(X-X_{0}\right)=\varnothing$. Since $X-f\left(X_{0}\right) \in \mathcal{B}$, applying again the fact that $f$ is an injection, we have that $\mu_{f}\left(X-f\left(X_{0}\right)\right)=\mu\left(f^{-1}\left(X-f\left(X_{0}\right)\right)\right)=\mu\left(f^{-1}(X)-f^{-1}\left(f\left(X_{0}\right)\right)\right)=\mu\left(X-X_{0}\right)=0$, i.e. $\mu_{f}\left(X-f\left(X_{0}\right)\right)=0$. But $B_{2} \subset X-X_{0}$, whence $f\left(B_{2}\right) \subset f\left(X-X_{0}\right)=$ $f(X)-f\left(X_{0}\right) \subset X-f\left(X_{0}\right)$, which consequently implies that $f\left(B_{2}\right) \in \mathcal{B}_{\mu}$.

We showed therefore that $f\left(B_{1}\right) \in \mathcal{B}_{\mu}$ and $f\left(B_{2}\right) \in \mathcal{B}_{\mu}$. Hence taking into account (1) we infer that also $f(B) \in \mathcal{B}_{\mu}$, and that is what we wished to prove.

Remark. Proposition 1 is also true without the assumption that $f$ is a one-to-one mapping, but only for the $\mu$-measurable sets of full measure (and only for $\sigma$-finite measures). This follows from the following statement.

Proposition 2. Assume that the measure $\mu$ is $\sigma$-finite, and let $f$ be a $\mu$-measurable mapping from $X$ into $X$. If $\mu \ll \mu_{f}$, then for every set $B \in \mathcal{B}_{\mu}$ such that $\mu(X-B)=$ 0 we have that $f(B) \in \mathcal{B}_{\mu}$ and $\mu(X-f(B))=0$.

In particular, if $\mu$ is a probability [finite] measure, then from $B \in \mathcal{B}_{\mu}$ and $\mu(B)=$ $1[\mu(B)=\mu(X)]$ it follows that $f(B) \in \mathcal{B}_{\mu}$ and $\mu(f(B))=1[\mu(f(B))=\mu(X)]$.

Proof. The proof will be divided into two steps. Suppose in the first place that the measure $\mu$ is finite. For simplicity we may obviously assume that $\mu$ is a probability measure, i.e. $\mu(X)=1$.

By virtue of Lemma 2 there exists a Borel subset $X_{0}$ of $X$ such that $\mu\left(X-X_{0}\right)=$ 0 , i.e. $\mu\left(X_{0}\right)=1$, and the restriction $\left.f\right|_{X_{0}}$ is Borel measurable.

Now let $B \in \mathcal{B}_{\mu}$ and $\mu(B)=1$. From the definition of the $\sigma$-algebra $\mathcal{B}_{\mu}$ we have that there is a Borel subset $B_{1}$ of $B$ such that $\mu\left(B_{1}\right)=1$. Put $B_{0}=B_{1} \cap X_{0}$. Then $B_{0} \in \mathcal{B}$ and $\mu\left(B_{0}\right)=1$. Since $B_{0} \subset X_{0}, X_{0} \in \mathcal{B}, \mu\left(X_{0}\right)=1$ and $\left.f\right|_{X_{0}}$ is a Borel mapping, we infer by virtue of the Lusin theorem (see [3, Corollary 24.22]) that for any $n=1,2, \ldots$ there exists a compact subset $K_{n}$ of $B_{0}$ such that $\mu\left(B_{0}-K_{n}\right)<1 / n$ and the restriction $\left.f\right|_{K_{n}}$ of $f$ to $K_{n}$ is a continuous mapping on $K_{n}$.

Put $X_{1}=\bigcup_{n=1}^{\infty} K_{n}$ and $Y_{1}=\bigcup_{n=1}^{\infty} f\left(K_{n}\right)$. It is clear that $X_{1} \in \mathcal{B}$ and $\mu\left(X_{1}\right)=$ 1. Moreover, since $f$ is a continuous mapping on $K_{n}$, then $f\left(K_{n}\right)$ is a compact set. Therefore $Y_{1}$ is a $\sigma$-compact and consequently a Borel subset of $X$, i.e. $Y_{1} \in \mathcal{B}$. Furthermore we have that

$$
f^{-1}\left(Y_{1}\right)=\bigcup_{n=1}^{\infty} f^{-1}\left(f\left(K_{n}\right)\right) \supset \bigcup_{n=1}^{\infty} K_{n}=X_{1} .
$$

Thus $f^{-1}\left(Y_{1}\right) \supset X_{1}$, whence $\mu_{f}\left(Y_{1}\right)=\mu\left(f^{-1}\left(Y_{1}\right)\right) \geq \mu\left(X_{1}\right)=1$. Therefore $\mu_{f}\left(Y_{1}\right)=1$, and taking into account that $\mu \ll \mu_{f}$ we obtain that $\mu\left(Y_{1}\right)=1$. Moreover, since for any $n=1,2, \ldots, K_{n} \subset B_{0}$, then $f\left(K_{n}\right) \subset f\left(B_{0}\right)$, and consequently $\bigcup_{n=1}^{\infty} f\left(K_{n}\right) \subset f\left(B_{0}\right)$, i.e. $Y_{1} \subset f\left(B_{0}\right)$. Hence $f\left(B_{0}\right) \in \mathcal{B}_{\mu}$ and $\mu\left(f\left(B_{0}\right)\right)=1$. But 
$B_{0} \subset B_{1} \subset B$, whence $f\left(B_{0}\right) \subset f(B)$. Therefore $f(B) \in \mathcal{B}_{\mu}$ and $\mu(f(B))=1$, which completes the first part of the proof, for the case when the measure $\mu$ is finite.

Suppose now that $\mu$ is an arbitrary $\sigma$-finite measure. This means that $X=$ $\bigcup_{n=1}^{\infty} X_{n}$, where for any $n=1,2, \ldots, X_{n} \in \mathcal{B}$ and $\mu\left(X_{n}\right)<\infty$. Let $B \in \mathcal{B}_{\mu}$ and $\mu(X-B)=0$. If we set $B_{n}=B \cap X_{n}$ (for $n=1,2, \ldots$ ), then $B_{n} \in \mathcal{B}_{n}$ and the fact that $X_{n}-B_{n} \subset X-B$ gives that $\mu\left(X_{n}-B_{n}\right)=0$. Since $B_{n} \subset X_{n}$ and $\mu\left(X_{n}\right)<\infty$, then identical considerations as in the first part of this proof show that $f\left(B_{n}\right) \in \mathcal{B}_{\mu}$ and $\mu\left(f\left(B_{n}\right)\right)=\mu\left(X_{n}\right)$, i.e. $\mu\left(X_{n}-f\left(B_{n}\right)\right)=0$. But $B=\bigcup_{n=1}^{\infty} B_{n}$, whence $f(B)=\bigcup_{n=1}^{\infty} f\left(B_{n}\right)$. Therefore also $f(B) \in \mathcal{B}_{\mu}$. Moreover $X-f(B)=\bigcup_{n=1}^{\infty} X_{n}-\bigcup_{n=1}^{\infty} f\left(B_{n}\right) \subset \bigcup_{n=1}^{\infty}\left(X_{n}-f\left(B_{n}\right)\right)$. Hence $\mu(X-f(B)) \leq$ $\sum_{n=1}^{\infty} \mu\left(X_{n}-f\left(B_{n}\right)\right)=0$, i.e. $\mu(X-f(B))=0$. The proposition is thus proved.

There is a question if the theorem inverse to Proposition 1 is also true. Now we show that such an inverse theorem is in reality true, if we make some additional assumptions about the measure $\mu$ and the mapping $f$. However, as opposed to Proposition 1 we do not assume that $f$ is an injection.

Proposition 3. Suppose that the measure $\mu$ is $\sigma$-finite and non-atomic, and $f$ is a $\mu$-mesurable mapping from $X$ onto $X$ (i.e. $f(X)=X$ ). If $f(B) \in \mathcal{B}_{\mu}$ for every set $B \in \mathcal{B}_{\mu}$, then $\mu \ll \mu_{f}$.

Proof. Suppose that our assertion is not true. This means that there exists a set $B_{1} \in \mathcal{B}$ such that $\mu_{f}\left(B_{1}\right)=0$ and $\mu\left(B_{1}\right)>0$. Then $f^{-1}\left(B_{1}\right) \in \mathcal{B}_{\mu}$ and from the definition of $\mu_{f}$ we have that $\mu\left(f^{-1}\left(B_{1}\right)\right)=0$. Since $B_{1} \in \mathcal{B}, \mu\left(B_{1}\right)>0$ and $\mu$ is a $\sigma$-finite and non-atomic measure, we get from Lemma 1 that there is a set $A_{1} \subset B_{1}$ which is not $\mu$-measurable (i.e. $\left.A_{1} \notin \mathcal{B}_{\mu}\right)$. Let $B=f^{-1}\left(A_{1}\right)$. Evidently $B \neq \varnothing$, which follows from the fact that $A_{1} \subset f(X)=X$. Since $A_{1} \subset B_{1}$, then $f^{-1}\left(A_{1}\right) \subset f^{-1}\left(B_{1}\right)$, i.e. $B \subset f^{-1}\left(B_{1}\right)$. But $f^{-1}\left(B_{1}\right) \in \mathcal{B}_{\mu}$ and $\mu\left(f^{-1}\left(B_{1}\right)\right)=0$, which implies that $B \in \mathcal{B}_{\mu}$. On the other hand we have however that $f(B)=f\left(f^{-1}\left(A_{1}\right)\right)=A_{1}$ (since $\left.f(X)=X\right)$. But $A_{1} \notin \mathcal{B}_{\mu}$, that is $f(B) \notin \mathcal{B}_{\mu}$. Therefore we have found a set $B \in \mathcal{B}_{\mu}$ such that $f(B) \notin \mathcal{B}_{\mu}$. But this contradicts the assumption and consequently completes the proof.

Remark. Proposition 3 is not true if we do not assume that $f$ is an onto mapping (even if we suppose that $f$ is an injection). This follows from the following example.

Let $X=[0,1]$ be a unit interval, $\mathcal{B}$ the Borel $\sigma$-algebra on $X$ and $\mu=m$ the Lebesgue measure on $\mathcal{B}$. We define a mapping $f:[0,1] \rightarrow[0,1]$ putting $f(x)=\frac{1}{2} x$, for $x \in[0,1]$. Obviously $f$ is a one-to-one Borel mapping but not a surjection (since $\left.f([0,1])=\left[0, \frac{1}{2}\right]\right)$. It is easy to check that $f(B) \in \mathcal{B}_{m}$ for every set $B \in \mathcal{B}_{m}$ (see [3], Th. 21.1), but it is not true that $\mu \ll \mu_{f}$. Indeed, if we put for example $B=\left(\frac{1}{2}, 1\right]$, then $\mu_{f}(B)=\mu\left(f^{-1}(B)\right)=\mu(\varnothing)=0$, but on the other hand $\mu(B)=\frac{1}{2}$. Therefore the assumption in Proposition 3 that $f$ is a surjection is in reality essential.

As a corollary from Propositions 1 and 3 we thus obtain the following theorem.

Theorem 1. Let $X$ be a separable complete metric space and $\mu$ a $\sigma$-finite nonatomic measure defined on the Borel $\sigma$-algebra $\mathcal{B}$ of the space $X$. Assume that $f$ is a one-to-one $\mu$-measurable mapping from $X$ onto $X$. Then the following two conditions are equivalent:

(a) $f(B) \in \mathcal{B}_{\mu}$ for every set $B \in \mathcal{B}_{\mu}$.

(b) $\mu \ll \mu_{f}$. 
At the end of this paper we shall prove some other properties of $\mu$-measurable mappings on a metric space $X$, similar to Propositions 1 and 3 , but concerning inverse images of $\mu$-measurable sets. It is possible to treat the facts that follow (Propositions 4 and 5) as a peculiar form of the Theorem of Kuratowski for inverse images.

Recall that by definition a mapping $f: X \rightarrow X$ is $\mu$-measurable if

$$
f^{-1}(B) \in \mathcal{B}_{\mu} \quad \text { for any set } B \in \mathcal{B} \text {. }
$$

There arises the question if in this definition we can exchange the condition $B \in \mathcal{B}$ by the condition $B \in \mathcal{B}_{\mu}$. In the other words there is the problem if for a $\mu$-measurable mapping $f: X \rightarrow X$ the property

$$
f^{-1}(B) \in \mathcal{B}_{\mu} \quad \text { for any set } B \in \mathcal{B}_{\mu}
$$

is true.

Unfortunately, this fact need not be true, even if $f$ is a Borel mapping. Indeed, in Example 2 of [5] we have shown, that if $\nu$ is a Gaussian probability measure on $R$ and $\mu=\nu \times \nu \times \ldots$ is the product measure on the linear metric space $X=R^{\infty}=R \times R \times \cdots$, then the $\mu$-completion $\mathcal{B}_{\mu}$ of the Borel $\sigma$-algebra $\mathcal{B}=\mathcal{B}\left(R^{\infty}\right)$ is not invariant under all translations. This means, that there exists a set $B \in \mathcal{B}_{\mu}$ and an element $x_{0} \in R^{\infty}$ such that $B+x_{0} \notin \mathcal{B}_{\mu}$. Now let $f: R^{\infty} \rightarrow R^{\infty}$ be a translation on $R^{\infty}$ given by the formula $f(x)=x-x_{0}$ for $x \in R^{\infty}$. Clearly, $f$ is a Borel mapping on $R^{\infty}$. Moreover, $f^{-1}(B)=\left\{x \in R^{\infty}: f(x) \in B\right\}=\left\{x \in R^{\infty}\right.$ : $\left.x-x_{0} \in B\right\}=\left\{x \in R^{\infty}: x \in B+x_{0}\right\}=B+x_{0}$. Therefore $f^{-1}(B) \notin \mathcal{B}_{\mu}$.

Nevertheless, it is easy to prove that the condition (3) will be true for a $\mu$ measurable mapping $f$ if we assume that the measure $\mu_{f}$ is absolutely continuous with respect to $\mu$ (i.e. that $\mu_{f} \ll \mu$ ).

We receive therefore the following assertion which is similar to Proposition 1. But as opposed to this proposition we don't need to assume that $f$ is an injection.

Proposition 4. Let $f$ be a $\mu$-measurable mapping from $X$ into $X$. If $\mu_{f} \ll \mu$, then $f^{-1}(B) \in \mathcal{B}_{\mu}$ for every set $B \in \mathcal{B}_{\mu}$.

Proof. Thus let $B \in \mathcal{B}_{\mu}$, i.e. $B=A \cup N$ where $A \in \mathcal{B}, N \subset A_{1}, A_{1} \in \mathcal{B}$ and $\mu\left(A_{1}\right)=0$. Then

$$
f^{-1}(B)=f^{-1}(A) \cup f^{-1}(N) .
$$

Since $A \in \mathcal{B}$ and $A_{1} \in \mathcal{B}$, then $f^{-1}(A) \in \mathcal{B}_{\mu}$ and $f^{-1}\left(A_{1}\right) \in \mathcal{B}_{\mu}$. Moreover $\mu\left(f^{-1}\left(A_{1}\right)\right)=\mu_{f}\left(A_{1}\right)$. But $A_{1} \in \mathcal{B}$ and $\mu\left(A_{1}\right)=0$. Hence, since $\mu_{f} \ll \mu$, we obtain that $\mu_{f}\left(A_{1}\right)=0$, and consequently $\mu\left(f^{-1}\left(A_{1}\right)\right)=0$. But $f^{-1}(N) \subset f^{-1}\left(A_{1}\right)$, which implies that $f^{-1}(N) \in \mathcal{B}_{\mu}$. Since also $f^{-1}(A) \in \mathcal{B}_{\mu}$, then from (4) we conclude that $f^{-1}(B) \in \mathcal{B}_{\mu}$, and that is what we had to prove.

The theorem inverse to the above proposition is also true, if we assume that the measure $\mu$ is $\sigma$-finite and non-atomic and $f$ is an injection. Thus we have the following fact, analogous to Proposition 3.

Proposition 5. Suppose that the measure $\mu$ is $\sigma$-finite and non-atomic and $f$ is a one-to-one $\mu$-measurable mapping from $X$ into $X$. If $f^{-1}(B) \in \mathcal{B}_{\mu}$ for every set $B \in \mathcal{B}_{\mu}$, then $\mu_{f} \ll \mu$.

Proof. Assume that our assertion is not true. Then there exists a Borel subset $B_{1}$ such that $\mu\left(B_{1}\right)=0$ and $\mu_{f}\left(B_{1}\right)>0$. 
In view of Lemma 2 the restriction $\left.f\right|_{X_{0}}$ is Borel measurable for some Borel set $X_{0}$ such that $\mu\left(X-X_{0}\right)=0$. Put $B_{0}=B_{1} \cap f\left(X_{0}\right)$. Then $B_{0} \subset f\left(X_{0}\right) \subset f(X)$. Moreover, from the Theorem of Kuratowski it follows that $f\left(X_{0}\right) \in \mathcal{B}$. Therefore also $B_{0} \in \mathcal{B}$, and since $B_{0} \subset B_{1}$, then $\mu\left(B_{0}\right)=0$.

Observe now that $\mu_{f}\left(X-f\left(X_{0}\right)\right)=\mu\left(f^{-1}\left(X-f\left(X_{0}\right)\right)\right)=\mu\left(f^{-1}(X)-f^{-1}\left(f\left(X_{0}\right)\right)\right)$ $=\mu\left(X-X_{0}\right)=0$, i.e. $\mu_{f}\left(X-f\left(X_{0}\right)\right)=0$.

Furthermore, from $B_{0} \cup\left(B_{1}-B_{0}\right)=B_{1}$ we have that $\mu_{f}\left(B_{0}\right)+\mu_{f}\left(B_{1}-B_{0}\right)=$ $\mu_{f}\left(B_{1}\right)$. Hence taking into account that $\mu_{f}\left(B_{1}-B_{0}\right)=0$ (since $B_{1}-B_{0} \subset X-$ $\left.f\left(X_{0}\right)\right)$, we conclude that $\mu_{f}\left(B_{0}\right)=\mu_{f}\left(B_{1}\right)$, whence in particular $\mu_{f}\left(B_{0}\right)>0$.

We have shown therefore that there exists a Borel subset $B_{0}$ of $X$ such that $B_{0} \subset f(X), \mu\left(B_{0}\right)=0$ and $\mu_{f}\left(B_{0}\right)>0$, i.e. $\mu\left(f^{-1}\left(B_{0}\right)\right)>0$.

Let $A=f^{-1}\left(B_{0}\right)$. Then $A \in \mathcal{B}_{\mu}$ and $\mu(A)>0$. In view of the definition of the $\sigma$-algebra $\mathcal{B}_{\mu}$ this means that there exists a set $A_{0} \in \mathcal{B}$ such that $A_{0} \subset A$ and $\mu\left(A_{0}\right)=\mu(A)>0$. Hence by virtue of Lemma 1 we obtain that there is a set $A_{1} \subset A_{0}$ (and consequently $A_{1} \subset A$ ) such that $A_{1} \notin \mathcal{B}_{\mu}$.

Now let $B=f\left(A_{1}\right)$. Since $A_{1} \subset A$, then $f\left(A_{1}\right) \subset f(A)$. But $f(A)=f\left(f^{-1}\left(B_{0}\right)\right)$ $=B_{0}$ (since $\left.B_{0} \subset f(X)\right)$. Hence $B \subset B_{0}$ and since $B_{0} \in \mathcal{B}$ and $\mu\left(B_{0}\right)=0$, then $B \in \mathcal{B}_{\mu}$. Therefore by virtue of the assumption we have that also $f^{-1}(B) \in \mathcal{B}_{\mu}$. But on the other hand $f^{-1}(B)=f^{-1}\left(f\left(A_{1}\right)\right)=A_{1}$ (since $f$ is an injection) and $A_{1} \notin \mathcal{B}_{\mu}$. Thus we get a contradiction, which finishes our proof.

Remark. Proposition 5 is false if $f$ is not an injection. Indeed, let for example $X=[0,1], \mathcal{B}$ be the Borel $\sigma$-algebra on $X$ and $\mu=m$ the Lebesgue measure on $\mathcal{B}$. If we put $f(x)=1$ for $x \in[0,1]$, then the assumptions of Proposition 4 are fulfilled. In fact, $f$ is obviously a $\mu$-measurable mapping (even Borel), and for any set $B \in \mathcal{B}_{\mu}$ we have that either $f^{-1}(B)=\varnothing$ (if $1 \notin B$ ) or $f^{-1}(B)=[0,1]$ (if $1 \in B$ ). Therefore $f^{-1}(B) \in \mathcal{B}_{\mu}$ always. But the condition $\mu_{f} \ll \mu$ is not satisfied, since for example $\mu(\{1\})=0$ and $\mu_{f}(\{1\})=1>0\left(\mu_{f}\right.$ is a probability measure concentrated at the point 1$)$.

As a corollary from Propositions 4 and 5 we obtain the following theorem (similar to Theorem 1, except for the assumption that $f$ is a surjection).

Theorem 2. Let $X$ be a separable complete metric space and $\mu$ a $\sigma$-finite nonatomic measure defined on the Borel $\sigma$-algebra $\mathcal{B}$ of the space $X$. Assume that $f$ is a one-to-one $\mu$-measurable mapping from $X$ into $X$. Then the following two conditions are equivalent:

(a) $f^{-1}(B) \in \mathcal{B}_{\mu}$ for every set $B \in \mathcal{B}_{\mu}$.

(b) $\mu_{f} \ll \mu$.

We finish the paper with the following corollary from our results. Namely, combining Propositions 1 and 3-5 we get the theorem which gives necessary and sufficient conditions in order that for every set $B \in \mathcal{B}_{\mu}, f(B) \in \mathcal{B}_{\mu}$ and $f^{-1}(B) \in \mathcal{B}_{\mu}$ for a $\mu$-measurable mapping $f$ on $X$.

Theorem 3. Let $X$ be a separable complete metric space and $\mu$ a measure defined on the Borel $\sigma$-algebra $\mathcal{B}$ of the space $X$. Assume that $f$ is a one-to-one and $\mu$ measurable mapping from $X$ into $X$. In order that $f(B) \in \mathcal{B}_{\mu}$ and $f^{-1}(B) \in \mathcal{B}_{\mu}$ for every set $B \in \mathcal{B}_{\mu}$ it is sufficient and, if $\mu$ is a non-atomic and $\sigma$-finite measure and $f$ is a surjection, it is also necessary that the measures $\mu_{f}$ and $\mu$ are equivalent (i.e. $\mu_{f} \sim \mu$ ). 


\section{REFERENCES}

1. K. Kuratowski, Topology I, Academic Press-PWN, New York-Warszawa, 1966. MR 36:840

2. K. R. Parthasarathy, Probability measures on metric spaces, Academic Press, New York, 1967. MR 37:2271

3. K. R. Parthasarathy, Introduction to probability and measure, Macmillan Co. of India, Dehli, 1977. MR 58:31322

4. M. Suslin, Sur une définition des ensembles mesurables B sans nombres transfinis, C. R. Acad. Sci. Paris 164 (1917), 89.

5. A. Wiśniewski, Theorem of Kuratowski-Suslin for measurable mappings, Proc. Amer. Math. Soc. 123 (1995), 1475-1479. CMP 95:07

Institute of Mathematics, Szczecin University, Ul. Wielkopolska 15, 70-451 Szczecin, POLAND

E-mail address: awisniew@uoo.univ.szczecin.pl 